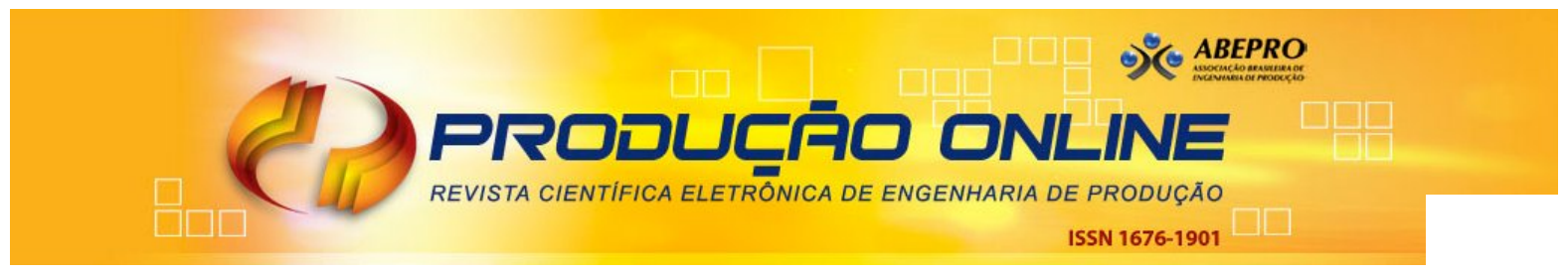

\title{
UMA REVISÃO SOBRE ABORDAGENS QUE RELACIONAM OS CUSTOS DE PRODUÇÃO E O PROCESSO DE PREVISÃO DE DEMANDA
}

\section{A LITERATURE REVIEW ON APPROACHES THAT RELATE THE PRODUCTION COSTS AND DEMAND FORECASTING}

\author{
Rodrigo Pessotto Almeida* E-mail: rodrigopa7@hotmail.com \\ Liane Werner* E-mail: liane@producao.ufrgs.br \\ *Universidade Federal do Rio Grande do Sul (UFRGS), Porto Alegre, RS
}

Resumo: Este artigo apresenta uma revisão da literatura sobre abordagens que relacionam os custos de produção e o processo de previsão de demanda. Esta revisão contempla artigos publicados em dezenove periódicos internacionais no período de 2002 a 2013. Os artigos avaliados correspondem a publicações localizadas nas bases de dados Web of Science e Scopus. O principal objetivo deste estudo consiste em classificar e analisar os artigos levantados de acordo com 7 abordagens identificadas. Como principais contribuições, este artigo apresenta uma revisão sobre abordagens que relacionam os conceitos de custos de produção e previsão de demanda no âmbito da produção, além de discussões acerca do tema, identificando a oportunidade de pesquisas futuras para abordagens como Planejamento de Operações e Planejamento de Capacidade.

Palavras chave: Custos de produção. Previsão de demanda. Produção. Planejamento de capacidade.

\begin{abstract}
This paper presents a literature review on approaches that relate the production costs and demand forecasting. The review comprises papers published from 2002 to 2013 in 19 international journals. The papers were obtained from Web of Science and Scopus data bases. The main objective was to conduct a classification and an analysis of the published papers according to 7 identified approaches. As main contributions, this article presents a revision about approaches that relate the concepts of production costs and demand forecast. In addition, the article shows the discussions on the subject, identifying suggestions for future researches on approaches like Operations Planning and Capacity Planning.
\end{abstract}

Keywords: Production costs. Demand forecasting. Manufacturing. Capacity planning.

\section{INTRODUÇÃO}

Dado o elevado nível de competição estabelecido pelas constantes mudanças dos cenários econômicos mundiais, inclusive no Brasil, surgem fatores que necessariamente devem receber atenção dos dirigentes de empresas. Neste contexto, fatores como o nível de desempenho em gerenciar, analisar e prever os custos do negócio caracterizam-se habilidades necessárias para estabelecer ou sustentar vantagens competitivas às organizações (SOBREIRO et al., 2008). 
Em tempos passados, com menor intensidade da concorrência, não se fazia necessária a busca contínua pela eficiência, justificado pelo fato do mercado absorver ineficiências e aceitar preços relativamente altos. Com a mudança deste cenário, passou a ser fundamental para as empresas a busca incessante por melhorias nos seus padrões de desempenho, que são representados pela eficiência e produtividade (BORNIA, 2010).

Neste sentido, uma nova abordagem dos sistemas de custos, a análise de custos de produção sob uma ótica gerencial, auxilia o controle e a avaliação da empresa, ao passo que consegue mensurar desperdícios e identificar atividades que não agregam valor ao produto. Assim, a transição dos atuais sistemas de custos de produção para sistemas voltados ao apoio gerencial ganha importância, pois apresentam condições de priorizar e dirigir esforços na busca pela excelência produtiva, constituindo assim uma poderosa ferramenta de apoio ao processo de tomada de decisão (BORNIA, 2010).

Outra maneira de auxiliar o processo de tomada de decisão é através da adoção de técnicas de previsão de demanda, que têm como finalidade fornecer informações oriundas do ambiente externo da empresa, básicas para o planejamento de suas atividades. As previsões de demanda de produtos e serviços permitem que os processos funcionais, logísticos, financeiros, marketing e de produção sejam adequadamente gerenciados, uma vez que podem ser previamente planejados (MAKRIDAKIS et al., 1998).

Em estudo apresentado por Danese e Kalchschimidt (2011a), os autores abordam o papel do processo de previsão de demanda em melhorar a acuracidade de suas previsões e também de aperfeiçoar o desempenho operacional nas organizações. Os autores apresentam como resultado desta pesquisa o impacto direto das variáveis do processo de previsão sobre o desempenho de custos da organização. Destacam também que a utilização de técnicas de previsão de demanda, bem como de diversas fontes de informações, apresentam positiva influência sobre o desempenho de custos.

Assim, previsões acuradas impactam diretamente em melhorias no desempenho de custos da organização, dado que viabilizam a redução de perdas. Com as informações subsidiadas pela previsão de demanda é possível, por meio da atividade de planejamento, estabelecer uma utilização otimizada de recursos, que 
por consequência fornecem maior competitividade à organização (DANESE e KALCHSCHIMIDT, 2011a; FISCHER e RAMAN, 1996; LI et al., 2013; PELLEGRINI e FOGLIATTO, 2001).

Conforme apresentado, é perceptível uma relação entre custos de produção e a previsão de demanda. Portanto, o principal objetivo deste artigo é realizar, por meio de um mapeamento da literatura no período de doze anos, uma classificação e análise dos artigos de acordo com as abordagens identificadas.

O presente artigo é subdividido em cinco seções. Após a introdução, na seção 2 são apresentados os procedimentos metodológicos utilizados na execução desta pesquisa. Na seção 3 é apresentada uma revisão da literatura, contemplando a classificação dos artigos mapeados na revisão sobre custos de produção e o processo de previsão de demanda. A análise dos artigos mapeados, que constitui os resultados, e as discussões estão apresentadas na seção 4. Por fim, na seção 5 são apresentadas as considerações finais do estudo.

\section{PROCEDIMENTOS METODOLÓGICOS}

Diversas são as classificações atribuídas aos diferentes métodos de pesquisa, empregados para formalizar a geração de conhecimento. Sampaio e Mancini (2007) definem revisão sistemática como uma forma de pesquisa que utiliza como fonte de dados a literatura existente de um determinado tema. Para os autores, esta forma de pesquisa constitui uma investigação que disponibiliza um resumo das evidências relacionadas a uma estratégia de intervenção, que é composta pelas seguintes etapas: aplicação de métodos explícitos e sistematizados de busca, apreciação crítica e síntese da informação selecionada. Utilizando este método de pesquisa, este trabalho foi desenvolvido conforme as seguintes etapas: (i) mapeamento dos artigos publicados na literatura, (ii) leitura e classificação dos artigos conforme suas abordagens e, (iii) análise de resultados.

Esta revisão iniciou através de uma busca em periódicos abrangidos pelas bases de dados: Web of Science e Scopus que mapeou um total de 731 artigos, sendo utilizado como critério de seleção os artigos que apresentavam a raiz das palavras "cost", "forecast" e "manufacturing" no título, resumo ou nas palavras chaves, publicados até o ano de 2013. A seguir optou-se por periódicos com um 
maior número de publicações sobre custos e previsão de demanda, ficando fora da seleção os periódicos com menos de três publicações sobre o assunto proposto e os periódicos não contemplados pelas bases de dados pesquisadas, reduzindo a um total de 238 publicações.

Como passo seguinte, adotou-se o critério de selecionar os artigos publicados nos anos que correspondem ao intervalo entre 2002 a 2013, o qual corresponde a uma recente e significativa parcela da literatura existente. Com o auxílio do software Mendeley, foi possível gerenciar os 156 artigos restantes, onde foi verificada a ocorrência de duplicidade para 38 artigos, por estarem publicados em ambas as bases pesquisadas, restando assim 118 publicações a serem submetidas ao critério final.

Como critério final, foram escolhidos os artigos com abordagem de análise de custos e previsão de demanda em sistemas de produção, identificada através da leitura dos abstracts, sendo necessária para alguns casos a leitura do texto completo.

Considerando apenas os artigos que atenderam a todos os critérios anteriormente determinados, incluindo à disponibilidade de acesso na íntegra de seus textos, 44 artigos foram selecionados. Porém a análise final foi conduzida através das abordagens apresentadas em 47 artigos, uma vez que 03 artigos constituem referências encontradas nos artigos selecionados pelos critérios acima descritos, e devido a sua relevância ao tema, no julgamento dos autores deste estudo, foram incluídos nesta revisão. Os artigos analisados constituem publicações localizadas nos periódicos apresentados no Quadro 1.

Após o mapeamento da bibliografia disponível, a segunda etapa envolveu a leitura e classificação das abordagens apresentadas. Com as abordagens apresentadas pelos artigos, foi possível identificar as seguintes classificações: Desempenho operacional, Estimativas de custos, Gestão de estoques, Planejamento da capacidade, Planejamento de operações, Planejamento de produção e, Cadeia de suprimentos.

Com a classificação dos artigos mapeados, na terceira etapa foi possível realizar a análise de resultados que sintetiza as informações obtidas com os artigos incluídos na revisão. Esta etapa contemplou a avaliação do número de artigos por 
periódico, número de artigos por ano de publicação, número de artigos por abordagem e oportunidades de pesquisas futuras.

Quadro 1 - Lista dos periódicos de publicação dos artigos mapeados

\begin{tabular}{|c|c|}
\hline \multicolumn{2}{|c|}{ Periódicos } \\
\hline $\begin{array}{l}\text { CIE } 2009 \text { International Conference on } \\
\text { Computers and Industrial Enqineering }\end{array}$ & Journal of Intelligent Manufacturing \\
\hline Computers and Industrial Engineering & $\begin{array}{l}\text { Journal of Manufacturing Science and } \\
\text { Engineering }\end{array}$ \\
\hline Computers and Operations Research & Journal of Operations Management \\
\hline $\begin{array}{l}\text { European Journal of Operational } \\
\text { Research }\end{array}$ & Journal Service Science \& Management \\
\hline $\begin{array}{l}\text { IEEE Transactions on Semicondutor } \\
\text { Manufacturing }\end{array}$ & Management Science \\
\hline Interfaces & $\begin{array}{l}\text { Manufacturing and Service Operations } \\
\text { Management }\end{array}$ \\
\hline $\begin{array}{l}\text { International Journal of Advanced } \\
\text { Manufacturing Technology }\end{array}$ & Mechanika \\
\hline $\begin{array}{l}\text { International Journal of Industrial } \\
\text { Engineering Theory Applications and } \\
\text { Practice }\end{array}$ & $\begin{array}{l}\text { Proceedings of the Institution of Mechanical } \\
\text { Engineers Part C: Journal of Mechanical } \\
\text { Engineering Science }\end{array}$ \\
\hline $\begin{array}{l}\text { International Journal of Production } \\
\text { Economics }\end{array}$ & Production Planning and Control \\
\hline $\begin{array}{l}\text { International Journal of Production } \\
\text { Research }\end{array}$ & \\
\hline
\end{tabular}

Fonte: Autores

\section{REVISÃO DA LITERATURA}

Com o surgimento de sistemas produtivos multiprodutores, o custeio da produção se tornou um princípio condutor para monitorar a eficiência de todas as atividades realizadas, bem como vincular 0 desempenho dos processos à rentabilidade global da organização (SOBREIRO e outros, 2008). Assim, o custeio da produção assume uma posição importante de apoio à tomada de decisão nas funções de gestão e controle das empresas.

Do mesmo modo, previsões de demanda desempenham importante papel na gestão organizacional, pois permitem o planejamento das diversas áreas, como área financeira, de recursos humanos e de vendas. Previsões também apresentam relação direta a diversos aspectos do gerenciamento de produção, como gestão de estoques e planejamento de produção (PELLEGRINI e FOGLIATTO, 2001).

De modo geral, as diversas técnicas de previsão de demanda podem ser classificadas em métodos quantitativos, qualitativos ou combinações de ambos. 
Métodos quantitativos geralmente são mais precisos, quando uma quantidade adequada de dados históricos esteja disponível e o fenômeno em investigação seja estável. Métodos qualitativos são utilizados quando não existem dados disponíveis e o fenômeno em investigação está em mudança, fazendo com que o passado seja incapaz de explicar o estado futuro (CANIATO et al., 2011).

Para Li et al. (2013), a obtenção de previsões mais precisas levam à redução dos custos totais de produção. Tais custos estão relacionados com a fabricação dos produtos, sendo normalmente divididos em Matéria-prima, Mão de obra direta e Custos indiretos de fabricação. Custos de matéria-prima correspondem aos principais materiais integrantes do produto. Os custos de mão de obra direta são aqueles relacionados aos trabalhadores diretamente envolvidos na fabricação dos produtos. Os demais custos de produção são classificados como custos indiretos de fabricação (BORNIA, 2010).

A classificação dos custos em termos de sua relação com o volume de produção divide-se em custos fixos e variáveis. Custos fixos são aqueles que independem do nível de atividade da empresa no curto prazo. Já os custos variáveis estão diretamente relacionados com o volume de produção (BORNIA, 2010).

Outra forma de classificação dos custos de produção apresentada pelo autor, considerando sua facilidade de alocação, é a separação destes em diretos e indiretos. Custos diretos são facilmente relacionados com as unidades de alocação de custos como, produtos e processos. Os custos indiretos não são facilmente atribuídos às unidades, exigindo a adoção de critérios para sua alocação.

Seguindo a relação de influência entre previsão de demanda e o desempenho de custos, gerados a partir da utilização dos recursos de produção, apresentada por Danese e Kalchschimidt (2011a), Fischer e Raman (1996), Li et al. (2013), Pellegrini e Fogliatto (2001), esta pesquisa buscou mapear artigos com abordagens que relacionam custos e previsão de demanda.

Com a leitura dos artigos selecionados, primeiramente identificaram-se as abordagens classificadas em 7 categorias, a saber: Cadeia de suprimentos, Desempenho operacional, Estimativas de custos, Gestão de estoques, Planejamento da capacidade, Planejamento de operações e Planejamento de produção. O Quadro 2 apresenta a classificação dos 47 artigos analisados, 
representados pela sua referência, identificando a distribuição destes em relação às 7 abordagens identificadas.

Quadro 2 - Abordagens identificadas e artigos em cada classificação

\begin{tabular}{|c|c|c|}
\hline Abordagens & \multicolumn{2}{|c|}{ Artigos } \\
\hline $\begin{array}{l}\text { Planejamento de } \\
\text { produção }\end{array}$ & $\begin{array}{l}\text { Khouja e Kumar (2002) } \\
\text { Lan e Lan (2005) } \\
\text { Chen et al. (2007) } \\
\text { Christou et al.(2007) } \\
\text { Briffaut e Lallement (2010) } \\
\text { Feng et al. (2011) }\end{array}$ & $\begin{array}{l}\text { Zhang et al. (2011) } \\
\text { Ponsignon e Lars (2012) } \\
\text { Tiacci e Saetta (2012) } \\
\text { Chhaochhria e Graves (2013) } \\
\text { Li et al. (2013) } \\
\text { Zhang et al. (2013) }\end{array}$ \\
\hline $\begin{array}{l}\text { Estimativas de } \\
\text { custos }\end{array}$ & $\begin{array}{l}\text { Cavalieri et al.(2004) } \\
\text { Tang et al. (2004) } \\
\text { Dai et al. (2006) } \\
\text { Bargelis e Rimašauskas (2007) } \\
\text { Wang (2007) }\end{array}$ & $\begin{array}{l}\text { Čikotiené e Bargelis (2009) } \\
\text { Chou et al. (2010) } \\
\text { Stasiškis et al. (2011) } \\
\text { Chang et al. (2012) } \\
\text { Stockton et al. (2013) }\end{array}$ \\
\hline $\begin{array}{l}\text { Desempenho } \\
\text { operacional }\end{array}$ & $\begin{array}{l}\text { Kassel e Tittmann (2007) } \\
\text { Chatfield e Hayya (2007) } \\
\text { Hillberg et al.(2009) } \\
\text { Wu et al. (2010) } \\
\text { Danese e Kalchschmidt (2011a) }\end{array}$ & $\begin{array}{l}\text { Danese e Kalchschmidt (2011b) } \\
\text { Rimašauskas e Bargelis (2012) } \\
\text { Agard e Bassetto (2013) } \\
\text { Tirkel (2013) }\end{array}$ \\
\hline $\begin{array}{l}\text { Cadeia de } \\
\text { suprimentos }\end{array}$ & $\begin{array}{l}\text { Thonemann (2002) } \\
\text { Cohen et al. (2003) } \\
\text { Ülkü et al. (2007) }\end{array}$ & $\begin{array}{l}\text { Yimer e Demirli (2010) } \\
\text { Kabak e Ulengin (2011) } \\
\text { Phuc et al. (2013) }\end{array}$ \\
\hline $\begin{array}{l}\text { Planejamento de } \\
\text { operações }\end{array}$ & $\begin{array}{l}\text { Saitou et al. (2002) } \\
\text { Meredith e Akinc (2007) }\end{array}$ & $\begin{array}{l}\text { Girardi e Camargo (2009) } \\
\text { Thome et al. (2012) }\end{array}$ \\
\hline $\begin{array}{l}\text { Gestão de } \\
\text { estoques }\end{array}$ & $\begin{array}{l}\text { Persona et al. (2007) } \\
\text { Saygin (2007) }\end{array}$ & Ji et al. (2008) \\
\hline $\begin{array}{l}\text { Planejamento da } \\
\text { capacidade }\end{array}$ & $\begin{array}{l}\text { Cakanyildirim e Roundy (2002) } \\
\text { Hood et al. (2003) }\end{array}$ & Bish et al. (2005) \\
\hline
\end{tabular}

Fonte: Autores

\subsection{PLANEJAMENTO DE PRODUÇÃO}

Com esta abordagem foram localizados doze artigos, onde a atividade de planejamento de produção é compreendida pela maioria dos autores como um grande desafio para organizações, em função de depender diretamente da capacidade destas em prever sua demanda, bem como ter impacto significativo nos custos de produção. Assim Chhaochhria e Graves (2013) abordam o planejamento da produção aplicando simulação para determinar políticas otimizadas de estoques que visa atender cenários de demanda incerta, assegurando alto nível de serviço aliado à minimização dos custos operacionais. Chen et al. (2007) apresentam quatro modelos para o planejamento baseados em previsão de demanda, custo de produção, capacidade produtiva e quantidade em estoque. Entendendo as dificuldades que a incerteza na demanda gera para o sistema de produção, Khouja e 
Kumar (2002) analisam investimentos e custos na aplicação de tecnologia da informação (TI) para tornar os sistemas de manufatura flexíveis, como forma de minimizar os problemas de planejamento de produção.

Briffaut e Lallement (2010) conduzem uma analogia desenvolvida através de conceitos e técnicas utilizadas no ambiente econômico, aplicando programação linear para definir o melhor momento para iniciar uma ordem de produção, tendo como variáveis dependentes o custo unitário e o preço de venda unitário, o qual é regulado pelo nível de demanda do mercado. Li et al. (2013), utilizando o algoritmo de aprendizado backpropagation neural network (BPNN), apresentam um modelo para previsão de demanda destinado a situações onde os dados disponíveis para condução de análises são limitados. Para um cenário com múltiplos produtos e componentes comuns, Zhang et al. (2013) apresentam um algoritmo que visa otimizar a eficiência do sistema de produção baseado no adiamento da diferenciação dos produtos, permitindo que informações de demanda do produto final sejam atualizadas próxima a etapa de sua fabricação.

Tiacci e Saetta (2012) apresentam três modelos simplificados utilizando Mixed Integer Programming (MIP), voltados a problemas de dimensionamento de lotes e programação da produção. Os modelos são aplicados em um estudo de caso real em uma empresa fabricante de pisos de madeira, onde custos de setup dependem da sequência programada de itens, a demanda futura é obtida a partir de previsões e o plano de produção é conduzido em horizonte rolante. Ponsignon e Lars (2012) desenvolvem um estudo para avaliar o problema de dimensionamento de recursos para atender demandas previstas, considerando as restrições de capacidade bem como os custos fixos relacionados. Como consequência, dois procedimentos heurísticos são propostos pelos autores: (i) produto baseado em esquemas de decomposição, e (ii) algoritmo genético. Com o objetivo de determinar o plano de produção que abrange exigências de cada período, minimizando todos os custos relevantes, Christou et al. (2007) apresentam uma abordagem de decomposição hierárquica do processo de planejamento de produção para multiprodutos. A aplicação parcial do modelo é conduzida em uma grande empresa engarrafadora de sucos e bebidas. 


\subsection{ESTIMATIVAS DE CUSTOS}

Nesta abordagem foram classificados dez artigos, onde o enfoque principal é direcionado à condução de estimativas de custos de produtos em fases iniciais de desenvolvimento. Dai et al. (2006) apresentam uma revisão metodológica que compreende o estado da arte relacionado às abordagens de estimativas de custos. Diversas técnicas e metodologias desenvolvidas ao longo dos anos são classificadas, discutindo suas principais vantagens e limitações, identificando tendências atuais e perspectivas futuras para o tema.

Wang (2007) descreve um processo de desenvolvimento de modelos de custos, além de conduzir uma avaliação sobre os efeitos de cada elemento estrutural de artificial neural networks (ANN), em relação à eficácia da modelagem de custos global em um ambiente de produção colaborativa. Cavalieri et al. (2004) apresentam um estudo onde são comparados os resultados alcançados com a aplicação de técnicas de estimativas tradicionais de custos com resultados obtidos através da implementação de um modelo utilizando ANN. Chou et al. (2010) trazem como proposta para estimativas de custos rápidas e precisas, um modelo baseado em inteligência artificial. Um modelo combinado para previsão de custo unitário do produto, que integra raciocínio baseado em casos (CBR) e ANN é proposto por Chang et al. (2012).

Para auxiliar os sistemas tradicionais de custos, Stockton et al. (2013) avaliam o desempenho de três algoritmos de mineração de dados com a finalidade de identificar e priorizar direcionadores de custos. Stasiškis et al. (2011) apresentam um modelo de classificação de produtos e componentes mecânicos, baseado em simulação, capaz de desenvolver estimativas de custos de produção. Já Čikotiené e Bargelis (2009) apresentam uma modelagem matemática capaz de prever custos de qualidade em processos de fabricação de produtos mecânicos, baseado em características como material, geometria, dimensão, tolerância e outros, auxiliando assim as empresas a minimizar perdas de recursos na operacionalização de seus processos. 


\subsection{DESEMPENHO OPERACIONAL}

Nesta categoria foram incluídos nove artigos, onde os principais autores discutem o impacto da acuracidade das previsões de demanda em relação ao desempenho operacional, avaliando principalmente o indicador custos de produção. Danese e Kalchschmidt (2011a) investigam os impactos da acuracidade das previsões e sua forma de condução sobre o desempenho operacional relacionado a custos de produção e entrega. Para estes a estrutura do processo de previsão deve ser coerente com a forma como os gestores pretendem aproveitar os resultados previstos, e com o objetivo que deve ser alcançado. Outro estudo apresentado pelos autores consiste na análise de hipóteses levantadas em relação à interpretação de como as variáveis do processo de previsão podem afetar o desempenho de custos e entrega da empresa (DANESE e KALCHSCHMIDT, 2011b).

Para Kassel e Tittmann (2007), a competitividade das organizações está vinculada a sua habilidade em compreender o comportamento de seus clientes, onde informações seguras sobre quantidades a serem produzidas em períodos futuros assegura a estas uma melhor otimização de seus processos produtivos, o que consequentemente, se traduz em redução dos custos de produção. Hillberg et al. (2009) convergem neste posicionamento, porém apresentam um estudo comparativo entre a técnica estatística de análise de regressão, ANN e técnicas de raciocínio baseado em casos (CBR), para construção de indicadores capazes de prever estados de desempenho futuro.

Para indústria de produtos tecnológicos, Wu et al. (2010) apresentam um modelo baseado na combinação de modelos difusos que reduzem a variância da previsão utilizando informações oriundas do mercado, adquiridas ao longo do tempo. A validação prática do modelo é conduzida em uma grande companhia fabricante de produtos eletrônicos, onde os resultados mostraram significativas reduções nos erros de suas previsões, provocando expressivas reduções de custos para a organização. Já Rimašauskas e Bargelis (2012) apresentam um modelo baseado em ANN com a finalidade de prever indicadores de produtividade na indústria de transformação.

Para decisões relacionadas ao projeto de novos produtos, Agard e Bassetto (2013) apresentam um algoritmo que busca otimizar requisitos de custos e 
qualidade. Em relação ao processo produtivo, Tirkel (2013) destaca o papel das curvas de aprendizagem em fornecer a base para melhorias na produtividade e em custos em diversas áreas de fabricação. Assim o autor investiga o desempenho destes modelos em um processo de fabricação de semicondutores.

\subsection{Cadeia de suprimentos}

Conforme os seis artigos mapeados nesta abordagem, muitos são os riscos associados às diversas operações da cadeia de suprimentos em um cenário de demanda incerta. Para lidar com estas incertezas, Phuc et al. (2013) utilizam programação dinâmica para determinar planos de produção otimizados em uma cadeia de suprimentos, considerando influências do mercado.

Cohen et al. (2003) investigam o problema enfrentado por fabricantes de produtos altamente personalizados de elevado valor econômico. Conforme apresentado pelos autores, estes fabricantes, previamente ao recebimento de um pedido formal, recebem de seus clientes, estimativas de potenciais demandas com a finalidade de melhorar o desempenho de toda a cadeia de suprimentos. Assim, existe o problema de definir o melhor momento para iniciar a mobilização da cadeia para atendimento à demanda, uma vez que existe trade-offs como a geração precipitada de custos aliada à possibilidade de cancelamento, ou elevação de custos em função de atraso. Para os autores, o simples compartilhamento de informações por si só não é suficiente para promover um desempenho otimizado da cadeia de suprimentos, sendo fundamental a predisposição de o cliente compartilhar os riscos juntamente com o fornecedor relativos à possibilidade de cancelamento de pedidos.

Já Ülkü et al. (2007) apontam a tendência das empresas em terceirizar parte de suas operações com a finalidade de se tornarem especialistas em seus processos. Assim eles discutem a distribuição dos riscos de produção relacionados à cadeia onde a demanda é incerta. Yimer e Demirli (2010) apresentam um estudo onde é utilizada programação dinâmica para o planejamento das atividades relacionadas à cadeia de suprimentos. No estudo conduzido por Kabak e Ulengin (2011), um modelo de programação linear é empregado para auxiliar no processo de tomada de decisão em relação ao planejamento de recursos, o qual utiliza informações como previsão de demanda, custos e capacidade. 


\subsection{Planejamento de operações}

Quatro artigos foram classificados nesta abordagem, destacando-se 0 trabalho conduzido por Thome et al. (2012), onde é apresentada uma revisão sistemática analisando 55 artigos sobre o Planejamento de Vendas e Operações (Sales and Operation Planning - S\&OP), constituindo a literatura disponível sobre este tema. Nesta abordagem, o S\&OP é analisado como uma ferramenta predominantemente tática, uma vez que integra o planejamento estratégico do negócio ao plano de operações, apresentando variações de acordo com os contextos industriais e estratégias de produção.

Meredith e Akinc (2007) apresentam uma nova estratégia de produção destinada a um segmento da indústria, como para fabricantes de equipamentos de engenharia de grande porte, que constitui um modelo híbrido que integra make-toorder (MTO) e make-to-stock (MTS), apresentando a estratégia chamada de maketo-forecast (MTF). Girardi e Camargo (2009) apresentam um modelo integrado de previsão utilizando a metodologia de Box \& Jenkins (modelos ARIMA) combinado com métodos qualitativos. Para validação este é comparado com um modelo baseado exclusivamente na experiência do pessoal da área de vendas, para a previsão de demanda de determinados produtos, na qual está baseado o planejamento de operações.

\subsection{Gestão de estoques}

A previsão de demanda assume um papel crucial, pois ao passo que as empresas buscam enfrentar incertezas de demanda necessariamente precisam controlar seus custos de produção, gerenciando corretamente seus níveis de estoque. Três artigos mapeados constituem abordagem sobre gestão de estoques, onde Persona et al. (2007) apresentam uma análise de custos baseada em modelos analíticos capazes de otimizar o nível de serviço prestado ao cliente em sistemas de produção Make to Order (MTO) e Assembly to Order (ATO). Estes modelos baseiam-se na determinação de níveis ótimos de estoques de segurança para conjuntos pré-montados e componentes, utilizados na fabricação do produto final. Já Saygin (2007) apresenta um comparativo entre modelos de gestão de estoques de produtos sensíveis ao tempo, com base em nível de serviço, custo, inventário, 
redução de perdas e complexidade na tomada de decisão. Além de dois modelos tradicionais baseados em níveis de estoque, é proposto um modelo de previsão de inventário formado pela integração de tecnologias de radio frequency identification (RFID) com técnicas de gestão de estoques em chão de fábrica. Ji et al. (2008) apresentam um modelo matemático com o objetivo de proporcionar o planejamento das atividades de transporte e dos níveis de inventário, a fim de minimizar custos relacionados.

\subsection{Planejamento de capacidade}

Foram encontrados três artigos orientados a planejamento de capacidade. Cakanyildirim e Roundy (2002), utilizando técnicas de planejamento de capacidade otimizadas e heurísticas, investigam alguns efeitos de práticas tradicionais no desempenho do planejamento de capacidade. Hood et al. (2003) conduzem um estudo aplicado ao planejamento de capacidade em indústrias fabricantes de semicondutores, apresentando um modelo para auxiliar na definição do número de ferramentas necessárias para o atendimento da demanda prevista, utilizando programação inteira estocástica para definir mudanças no perfil de demanda. Um conjunto de cenários de demanda discretos é considerado, gerando informações para aquisição de ferramentas sob uma restrição orçamentária, minimizando efeitos de demandas insatisfeitas. Bish et al. (2005) investigam os custos e benefícios associados à gestão da flexibilidade de capacidade para mitigar flutuações de curto prazo na demanda utilizando variadas políticas de alocação desta aos produtos.

\section{RESULTADOS E DISCUSSÃO}

Na Tabela 1 é apresentada uma análise da distribuição dos artigos mapeados por periódicos e ano de publicação. Observa-se que os periódicos International Journal of Production Economics, International Journal of Production Research e Journal of Intelligent Manufacturing concentram aproximadamente 42,6\% das publicações referentes a abordagens que relacionam custos de produção com 0 processo de previsão de demanda.

Em relação ao número de publicações ao longo dos anos, nota-se na Tabela 1, o maior pico de ocorrência no ano de 2007 com quase 21,3\% dos artigos 
publicados, e uma retomada do tema nos anos de 2011, 2012 e 2013, sendo estes responsáveis por concentrar aproximadamente 38,3\% dos artigos analisados.

A Tabela 2 apresenta uma análise dos artigos mapeados classificados de acordo com as abordagens identificadas e ao ano de publicação. Com base nesta classificação, os artigos estão distribuídos em sete abordagens ao longo de doze anos, com destaque para as abordagens Planejamento de Produção, Estimativas de Custos, e Desempenho Operacional, que concentram aproximadamente $66 \%$ dos artigos avaliados.

Tabela 1 - Número de artigos em relação aos periódicos e ao ano de publicação.

\begin{tabular}{|c|c|c|c|c|c|c|c|c|c|c|c|c|c|c|}
\hline Periódicos & §ั & ஜ̊̀ & ర్ & 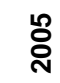 & \& & 定 & $\stackrel{\infty}{\circ}$ & 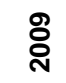 & 옴 & ন্ড & ָั่ & $\stackrel{m}{\stackrel{N}{N}}$ & Total & $\begin{array}{c}\% \\
\text { Acum. }\end{array}$ \\
\hline $\begin{array}{l}\text { International Journal of } \\
\text { Production Economics }\end{array}$ & & & 1 & & & 2 & & & 1 & 2 & 2 & 1 & 9 & 19,1 \\
\hline $\begin{array}{l}\text { International Journal of } \\
\text { Production Research }\end{array}$ & 1 & & & & & 2 & & & & 2 & & 2 & 7 & 34,0 \\
\hline $\begin{array}{l}\text { Journal of Intelligent } \\
\text { Manufacturing }\end{array}$ & 1 & & & & & 1 & & & & 1 & 1 & & 4 & 42,5 \\
\hline $\begin{array}{l}\text { International Journal of } \\
\text { Advanced Manufacturing } \\
\text { Technology }\end{array}$ & & & & 1 & & 1 & & & & & 1 & & 3 & 48,9 \\
\hline Management Science & & 1 & 1 & 1 & & & & & & & & & 3 & 55,3 \\
\hline Mechanika & & & & & & & & 1 & & 1 & 1 & & 3 & 61,7 \\
\hline $\begin{array}{l}\text { IEEE Transactions on } \\
\text { Semicondutor } \\
\text { Manufacturing }\end{array}$ & 1 & 1 & & & & & & & & & & 1 & 3 & 68,1 \\
\hline $\begin{array}{l}\text { Computers and } \\
\text { Industrial Engineering }\end{array}$ & & & & & & & & & 1 & & & 1 & 2 & 72,4 \\
\hline $\begin{array}{l}\text { International Journal of } \\
\text { Industrial Engineering } \\
\text { Theory Applications and } \\
\text { Practice }\end{array}$ & & & & & & & 1 & 1 & & & & & 2 & 76,7 \\
\hline $\begin{array}{l}\text { European Journal of } \\
\text { Operational Research }\end{array}$ & 1 & & & & & & & & & 1 & & & 2 & 81,0 \\
\hline $\begin{array}{l}\text { CIE } 2009 \text { International } \\
\text { Conference on } \\
\text { Computers and } \\
\text { Industrial Engineering }\end{array}$ & & & & & & & & 1 & & & & & 1 & 83,2 \\
\hline $\begin{array}{l}\text { Computers and } \\
\text { Operations Research }\end{array}$ & & & & & & & & & & & 1 & & 1 & 85,3 \\
\hline Interfaces & & & & & & & & & 1 & & & & 1 & 87,4 \\
\hline $\begin{array}{l}\text { Journal of Operations } \\
\text { Management }\end{array}$ & & & & & & 1 & & & & & & & 1 & 89,5 \\
\hline $\begin{array}{l}\text { Journal Service Science } \\
\& \text { Management }\end{array}$ & & & & & & & & & 1 & & & & 1 & 91,6 \\
\hline $\begin{array}{l}\text { Manufacturing and } \\
\text { Service Operations } \\
\text { Management }\end{array}$ & & & & & & 1 & & & & & & & 1 & 93,7 \\
\hline $\begin{array}{l}\text { Journal of } \\
\text { Manufacturing Science } \\
\text { and Engineering }\end{array}$ & & & & & 1 & & & & & & & & 1 & 95,8 \\
\hline $\begin{array}{l}\text { Proc. of the Institution of } \\
\text { Mechanical Engineers } \\
\text { Part C: Journal of } \\
\text { Mechanical Engineering } \\
\text { Science }\end{array}$ & & & & & & 1 & & & & & & & 1 & 97,9 \\
\hline $\begin{array}{l}\text { Production Planning and } \\
\text { Control }\end{array}$ & & & & & & 1 & & & & & & & 1 & 100,0 \\
\hline Total & 4 & 2 & 2 & 2 & 1 & 10 & 1 & 3 & 4 & 6 & 5 & 7 & 47 & 100 \\
\hline$\%$ & 8,5 & 4,3 & 4,3 & 4,3 & 2,1 & 21,3 & 2,1 & 6,4 & 8,5 & 12,8 & 10,6 & 14,9 & 100 & \\
\hline
\end{tabular}

Fonte: Autores 
Com a leitura dos 47 artigos sobre abordagens relacionadas ao tema, custos de produção e o processo de previsão de demanda, constatou-se a flexibilidade na utilização destes conceitos, bem como a diversidade de situações onde estes são empregados no ambiente de produção. Tais conceitos tem se tornado útil para a criação de estratégias de produção buscando a otimização na utilização e aplicação de recursos, por meio de atividades de planejamento e gestão operacional. Estes também são empregados em aplicações diversas voltadas à avaliação de desempenho dos sistemas produtivos.

Tabela 2 - Número de artigos em relação ao ano de publicação e às abordagens.

\begin{tabular}{|c|c|c|c|c|c|c|c|c|c|c|c|c|c|c|}
\hline Abordagens & ণั & ஜั & ষ্ণ & 농 & \& & 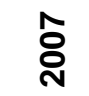 & $\stackrel{\text { ̊ }}{\circ}$ & ঃํ & 옹 & ন্் & సั่ & $\stackrel{m}{\stackrel{N}{N}}$ & Total & $\begin{array}{c}\% \\
\text { (acum.) }\end{array}$ \\
\hline $\begin{array}{l}\text { Planejamento } \\
\text { de produção }\end{array}$ & 1 & & & 1 & & 2 & & & 1 & 2 & 2 & 3 & 12 & 25,5 \\
\hline $\begin{array}{l}\text { Estimativas } \\
\text { de custos }\end{array}$ & & & 2 & & 1 & 2 & & 1 & 1 & 1 & 1 & 1 & 10 & 46,8 \\
\hline $\begin{array}{l}\text { Desempenho } \\
\text { operacional }\end{array}$ & & & & & & 2 & & 1 & 1 & 2 & 1 & 2 & 9 & 65,9 \\
\hline $\begin{array}{l}\text { Cadeia de } \\
\text { suprimentos }\end{array}$ & 1 & 1 & & & & 1 & & & 1 & 1 & & 1 & 6 & 78,7 \\
\hline $\begin{array}{l}\text { Planejamento } \\
\text { de operações }\end{array}$ & 1 & & & & & 1 & & 1 & & & 1 & & 4 & 87,2 \\
\hline $\begin{array}{l}\text { Gestão de } \\
\text { estoques }\end{array}$ & & & & & & 2 & 1 & & & & & & 3 & 93,6 \\
\hline $\begin{array}{l}\text { Planejamento } \\
\text { da } \\
\text { capacidade }\end{array}$ & 1 & 1 & & 1 & & & & & & & & & 3 & 100,0 \\
\hline $\begin{array}{c}\text { Total } \\
\%\end{array}$ & $\begin{array}{c}4 \\
8,5\end{array}$ & $\begin{array}{c}2 \\
4,3\end{array}$ & $\begin{array}{c}2 \\
4,3\end{array}$ & $\begin{array}{c}2 \\
4,3\end{array}$ & $\begin{array}{c}1 \\
2,1\end{array}$ & $\begin{array}{c}10 \\
21,3\end{array}$ & $\begin{array}{c}1 \\
2,1\end{array}$ & $\begin{array}{c}3 \\
6,4\end{array}$ & $\begin{array}{c}4 \\
8,5\end{array}$ & $\begin{array}{c}6 \\
12,8\end{array}$ & $\begin{array}{c}5 \\
10,6\end{array}$ & $\begin{array}{c}7 \\
14,9\end{array}$ & $\begin{array}{c}47 \\
100\end{array}$ & 100 \\
\hline
\end{tabular}

Fonte: Autores

É possível destacar as abordagens: Planejamento de Produção e Estimativas de Custos, por concentrarem aproximadamente $47 \%$ dos artigos mapeados. Para suportar a complexidade do ambiente de produção moderno, modelagens de custos tornam-se indispensáveis como ferramentas que podem produzir respostas mais precisas e flexíveis para a tomada de decisão (WANG, 2007). Diversas foram as abordagens conduzidas com a finalidade de estimar custos, porém, destaca-se a utilização por vários autores da técnica de ANN para esta atividade. No estudo conduzido por Dai et al. (2006), são apresentadas as diversas técnicas e metodologias desenvolvidas ao longo dos anos, discutindo suas principais vantagens e limitações, bem como a importância deste tema para as organizações. Fundamentalmente, as aplicações destes modelos se limitam a estimar custos de fabricação em fases iniciais do desenvolvimento de produto. 
Um posicionamento comum entre as abordagens de Planejamento de Produção é a complexidade de execução desta atividade provocada pela incerteza na demanda de produtos ou serviços a que as organizações estão submetidas (CHEN et al., 2007; KHOUJA e KUMAR, 2002; Ll et al., 2013). Diferentes técnicas foram identificadas para a atividade de planejar a produção, como modelos matemáticos, capacidade de flexibilização da manufatura e modelagem computacional. Todos os modelos apresentados baseiam-se na previsão de demanda como meio de planejar a necessidade de recursos, de tal forma a proporcionar redução nos custos relacionados à utilização destes recursos.

$\mathrm{Na}$ abordagem de Desempenho Operacional, evidencia-se a progressiva preocupação com questões relacionadas ao papel do processo de previsão de demanda sobre o desempenho do sistema produtivo. Para Hillberg et al. (2009), previsões acuradas acerca do estado futuro constituem informações úteis para os gestores na busca pela otimização dos sistemas produtivos. Nos estudos de Danese e Kalchschmidt (2011a) e Danese e Kalchschmidt (2011b) também é discutida a relação da acuracidade da previsão em relação a indicadores organizacionais, incluindo investigações sobre variáveis presentes neste processo.

Uma importante observação é o fato de uma significativa tendência em incorporar aspectos financeiros e parâmetros econômicos nas diversas abordagens que relacionam previsão de demanda e avaliação de custos, buscando formalizar ferramentas de auxílio à tomada de decisão. Neste sentido, estas se tornam técnicas com linguagem facilmente entendidas pelos gestores e demais usuários.

Conforme apresentado por Lan e Lan (2005), pesquisas com foco no planejamento de recursos de produção em diferentes cenários representam uma importante frente de pesquisa, uma vez que esta pode ser considerada uma atividade estratégica na maioria dos sistemas produtivos.

Conforme proposto por Thome et al. (2012), a integração de planos financeiros junto ao processo de Planejamento de Vendas e Operações (S\&OP) constitui uma importante linha de pesquisa, uma vez que existem poucos artigos que realizam esta abordagem, incluindo o fato desta ser uma necessidade apontada por diversos pesquisadores, ressalta o autor. Adicionalmente observa-se que a inclusão de parâmetros econômicos nesta análise pode trazer benefícios às organizações, 
pois o S\&OP tem como objetivo melhorar resultados, otimizando receitas e lucros, estabelecendo um equilíbrio entre oferta, capacidade dísponivel e demanda.

Para o tópico de estimativas de custos, verifica-se a oportunidade de ampliação de estudos relacionados à utilização de técnicas recentes como inteligência artificial, CBR e ANN para alcançar estimativas de custos rápidas e precisas, auxiliando o processo de tomada de decisão. Estudos de casos aplicados à indústria, utilizando estes conceitos, também contituem interessantes linhas de pesquisa.

Corroborando com as abordagens apresentadas por Persona et al. (2007) e Bish et al. (2005), estudos voltados à adoção de conceitos de gestão da flexibilidade da capacidade e gestão de estoques, caracterizam significativas linhas de pesquisa para otimização de custos na configuração e operação dos sistemas produtivos.

Considerando a parcela da literatura abrangida por este estudo, identifica-se que a abordagem de planejamento de capacidade tem sido objeto de poucas publicações. Para Yimer e Demirli (2010), no atual ambiente competitivo, agilidade e processos enxutos se tornaram preocupações estratégicas para empresas de manufatura em seus esforços para ampliar sua participação de mercado. Assim, verifica-se a necessidade de estudos que discutam a avaliação da capacidade instalada de um sistema produtivo frente à capacidade economicamente interessante para atendimento da demanda de mercado. Esta análise é interessante para as empresas de manufatura, pois permite avaliar de forma pró ativa o nível de alinhamento de sua capacidade produtiva com o seu mercado.

\section{CONSIDERAÇÕES FINAIS}

Abordagens que relacionam o processo de previsão de demanda com a avaliação de custos de produção podem revelar importantes medidas de apoio à gestão empresarial, bem como auxiliar na otimização dos sistemas produtivos, uma vez que gerenciar, analisar e prever custos do negócio é essencial para estabelecer vantagens competitivas para a organização (SOBREIRO et al., 2008). O presente estudo levantou artigos sobre abordagens relacionadas ao tema, custos de produção e o processo de previsão de demanda no âmbito de produção, publicados no período de 2002 a 2013. Com este levantamento foram identificados e analisados 47 artigos, posteriormente classificados em 7 abordagens. Adicionalmente foram 
realizadas análises em relação à distribuição destes artigos, considerando publicações por ano, artigos por periódicos e número de artigos por abordagem.

Constata-se que os periódicos International Journal of Production Economics, International Journal of Production Research e Journal of Intelligent Manufacturing concentram aproximadamente $42,6 \%$ do total das publicações. Em relação aos tópicos de interesse, verifica-se que as abordagens Planejamento de Produção, Estimativas de Custos, e Desempenho Operacional concentram aproximadamente $66 \%$ do total de artigos avaliados.

O tópico Estimativas de Custos assume um papel crucial nas organizações, uma vez que apresenta relação direta no desempenho e eficácia destas, onde superestimação de custos pode resultar em perda de negócios, enquanto que a subestimação pode levar a significativas perdas financeiras para a empresa (DAl et al., 2006).

Segundo Chen et al. (2007), Khouja e Kumar (2002) e Li et al. (2013), a incerteza da demanda de produtos ou serviços faz com que o planejamento de produção seja uma atividade relativamente complexa para as organizações. Assim, visando a otimização dos sistemas produtivos, tornan-se indispensáveis a adoção de modelos que forneçam previsões acuradas acerca do estado futuro. Do mesmo modo, o Desempenho Operacional também estará condicionado ao nível de acuracidade das previsões, pois consiste na execução do planejamento.

Outra forma de enfrentar este problema é o emprego de medidas de flexibilização da capacidade produtiva (BISH et al., 2005). Já Persona et al. (2007), Chhaochhria e Graves (2013) destacam a utilização de políticas de gestão de estoques como alternativa para lidar com incertezas na demanda.

Entretanto, estudos relacionados às abordagens de Planejamento de Operações e Planejamento de Capacidade ainda apresentam oportunidades de maiores pesquisas, sendo oportuna a incorporação de conceitos adicionais, como econômicos e financeiros. Conforme Silva e Leite (2013), são necessários estudos sobre planejamento de capacidade de longo prazo, apoiados em previsões de demanda acuradas, com o intuito de garantir a plena utilização dos recursos. Estes destacam também a necessidade de um controle de custos contínuo, para atingir custos cada vez mais baixos, visando à satisfação dos clientes. 
Portanto, é evidente a contribuição que decisões, fundamentadas em custos e previsão de demanda, podem fornecer às organizações em seu desafio de equilibrar capacidade de produção com a demanda.

Como principais contribuições do presente artigo destacam-se: (i) identificação de significativa parcela de abordagens existentes sobre o tema proposto; (ii) classificação dos artigos avaliados em relação a sua abordagem; e (iii) análise da classificação, identificando oportunidades de pesquisas futuras, sendo útil para pesquisadores voltados à área de otimização de sistemas produtivos.

\section{REFERÊNCIAS}

AGARD, B.; BASSETTO, S. Modular design of product families for quality and cost. International Journal of Production Research, v. 51, $6^{\text {th }}$ ed., p. 1648-1667, 2013. http://dx.doi.org/10.1080/00207543.2012.693963

BARGELIS, A.; RIMAŠAUSKAS, M. Cost forecasting model for order-based sheet metalworking. Proceedings of the Institution of Mechanical Engineers, Part C: Journal of Mechanical Engineering Science, v. 221(1), p. 55-65, 2007.

http://dx.doi.org/10.1243/0954406JMES269

BISH, E. K.; MURIEL, A.; BILLER, S. Managing flexible capacity in a make-to-order environment. Management Science, v. 51(2), p. 167-18, 2005.

http://dx.doi.org/10.1287/mnsc.1040.0322

BORNIA, A. C. Análise gerencial de custos: aplicação em empresas modernas. 3. ed. São Paulo: Atlas, 2010.

BRIFFAUT, J.-P.; LALLEMENT, P. Volatility Forecasting of Market Demand as Aids for Planning Manufacturing Activities. Journal Service Science \& Management, v. 3, p. 383389, 2010. http://dx.doi.org/10.4236/jssm.2010.34045

CAKANYILDIRIM, M.; ROUNDY, R. O. Evaluation of capacity planning practices for the semiconductor industry. IEEE Transactions on Semiconductor Manufacturing, v. 15 (3), p. 331-340, 2002. http://dx.doi.org/10.1109/TSM.2002.801386

CANIATO, F.; KALCHSCHMIDT, M.; RONCHI, S. Integrating quantitative and qualitative forecasting approaches: organizational learning in an action research case. Journal of the Operational Research Society, v. 62, p. 413-424, 2011.

http://dx.doi.org/10.1057/jors.2010.142

CAVALIERI, S.; MACCARRONE, P.; PINTO, R. Parametric vs. neural network models for the estimation of production costs: A case study in the automotive industry. International Journal of Production Economics, v. 91, p. 165-177, 2004.

http://dx.doi.org/10.1016/j.ijpe.2003.08.005

CHANG, P.-C.; LIN, J.-J.; DZAN, W.-Y. Forecasting of manufacturing cost in mobile phone products by case-based reasoning and artificial neural network models. Journal of Intelligent Manufacturing, v. 23 (3), p. 517-531, 2012. 
http://dx.doi.org/10.1007/s10845-010-0390-7

CHATFIELD, D. C.; HAYYA, J. C. All-zero forecasts for lumpy demand: a factorial study. International Journal of Production Research, v. 45 (4), p. 935-950, 2007. http://dx.doi.org/10.1080/00207540600622480

CHEN, A.; HSU, C.-H.; BLUE, J. Demand planning approaches to aggregating and forecasting interrelated demands for safety stock and backup capacity planning. International Journal of Production Research, v. 45 (10), p. 2269-2294, 2007. http://dx.doi.org/10.1080/00207540600690693

CHHAOCHHRIA, P.; GRAVES, S. C. A forecast-driven tactical planning model for a serial manufacturing system. International Journal of Production Research, v. 51, n. 23-24, p. 6860-6879, 2013. http://dx.doi.org/10.1080/00207543.2013.852266

CHOU, J.; TAI, Y.; CHANG, L. Predicting the development cost of TFT-LCD manufacturing equipment with artificial intelligence models. International Journal of Production Economics, v. 128 (1), p. 339-350, 2010. http://dx.doi.org/10.1016/j.ijpe.2010.07.031

CHRISTOU, I. T.; LAGODIMOS, A. G.; LYCOPOULOU, D. Hierarchical production planning for multi-product lines in the beverage industry. Production Planning \& Control, v. 18 (5), p. 367-376, 2007. http://dx.doi.org/10.1080/09537280701340631

ČIKOTIENĖ, D.; BARGELIS, A. Process modeling for quality in order-handled manufacturing system. Mechanika, v. 1 (1), p. 47-55, 2009.

COHEN, M. A.; HO, T. H.; REN, Z. J.; TERWIESCH, C. Measuring imputed cost in the semiconductor equipment supply chain. Management Science, v. 49 (12), p. 1653-1670, 2003. http://dx.doi.org/10.1287/mnsc.49.12.1653.25115

DAI, J. S.; NIAZI, A.; BALABANI, S.; SENEVIRATNE, L. Product Cost Estimation: Technique Classification and Methodology Review. Journal of Manufacturing Science and

Engineering, v. 128, p. 563-575, 2006. http://dx.doi.org/10.1115/1.2137750

DANESE, P.; KALCHSCHMIDT, M. The impact of forecasting on companies' performance : Analysis in a multivariate setting. International Journal of Production Economics, v. 133 (1), p. 458-469, 2011b. http://dx.doi.org/10.1016/.ijpe.2010.04.016

DANESE, P.; KALCHSCHMIDT, M. The role of the forecasting process in improving forecast accuracy and operational performance. International Journal of Production Economics, v. 131 (1), p. 204-214, 2011a. http://dx.doi.org/10.1016/j.ijpe.2010.09.006

FENG, P.; ZHANG, J.; WU, Z.; YU, D. An improved production planning method for process industries. International Journal of Production Research, v. 49 (14), p. 4223-4243, 2011. http://dx.doi.org/10.1080/00207543.2010.547528

FISCHER, M.; RAMAN, A. Reducing the cost of demand uncertainty through accurate response to early sales. Operations Research, v. 44, p. 87-99, 1996.

http://dx.doi.org/10.1287/opre.44.1.87

GIRARDI, G.; CAMARGO, M. E. Forecast Production Volume: A Case Study. CIE: 2009 International Conference on Computers and Industrial Engineering, v. 1-3, p. 17471750, 2009. http://dx.doi.org/10.1109/ICCIE.2009.5223501 
HILLBERG, P. A.; SENGUPTA, S.; VAN TIL, R. P. A Comparative Study of Three Predictive Tools for Forecasting a Transfer Line's Throughput. International Journal of Industrial Engineering-Theory Applications and Practice, v. 16 (1), p. 32-40, 2009.

HOOD, S. J.; BERMON, S.; BARAHONA, F. Capacity planning under demand uncertainty for semiconductor manufacturing. IEEE Transactions on Semiconductor Manufacturing, $v$. 16 (2), p. 273-280, 2003. http://dx.doi.org/10.1109/TSM.2003.811894

JI, P.; CHEN, K. J.; YAN, Q. P. A Mathematical Model for a Multi-Commodity, Two-Stage Transportation and Inventory Problem. International Journal of Industrial Engineering Theory Applications and Pratice, v. 15 (3), p. 278-285, 2008.

KABAK, O.; ULENGIN, F. Possibilistic linear-programming approach for supply chain networking decisions. European Journal of Operational Research, v. 209 (3), p. 253-264, 2011. http://dx.doi.org/10.1016/j.ejor.2010.09.025

KASSEL, S.; TITTMANN, C. Implications from customer behavior for manufacturing. Journal of Intelligent Manufacturing, v. 18 (4), p. 475-478, 2007.

http://dx.doi.org/10.1007/s10845-007-0050-8

KHOUJA, M.; KUMAR, R. L. Information technology investments and volume-flexibility in production systems. International Journal of Production Research, v. 40 (1), p. 205-221, 2002. http://dx.doi.org/10.1080/00207540110072948

LAN, C.-H.; LAN, T.-S. A combinatorial manufacturing resource planning model for long-term CNC machining industry. International Journal of Advanced Manufacturing Technology, v. 26 (9-10), p. 1157-1162, 2005. http://dx.doi.org/10.1007/s00170-004-2090-y

LI, D.-C.; CHANG, C.-C.; LIU, C.-W.; CHEN, W.-C. A new approach for manufacturing forecast problems with insufficient data: the case of TFT-LCDs. Journal of Intelligent Manufacturing, p. 225-233, 2013. http://dx.doi.org/10.1007/s10845-011-0577-6

MAKRIDAKIS, S.; WHEELWRIGHT, S. C.; HYNDMAN, R. J. Forecasting: methods and applications. $3^{\text {rd }}$ ed. New York: John Wiley \& Sons, 1998.

MEREDITH, J.; AKINC, U. Characterizing and structuring a new make-to-forecast production strategy. Journal of Operations Management, v. 25 (3), p. 623-642, 2007.http://dx.doi.org/10.1016/j.jom.2006.04.006

PELLEGRINI, F. R.; FOGLIATTO, F. S. Passos para Implantação de Sistemas de Previsão de Demanda - Técnicas e Estudo de Caso. Revista Produção, v. 11, n.1, p. 43-64, 2001.http://dx.doi.org/10.1590/S0103-65132001000100004

PERSONA, A.; BATTINI, D.; MANZINI, R.; PARESCHI, A. Optimal safety stock levels of subassemblies and manufacturing components. International Journal of Production Economics, v. 110 (1-2), p. 147-159, 2007. http://dx.doi.org/10.1016/j.ijpe.2007.02.020

PHUC, P. N. K.; YU, V. F.; CHOU, S.-Y. Manufacturing production plan optimization in threestage supply chains under Bass model market effects. Computers \& Industrial Engineering, v. 65, p. 509-516, 2013. http://dx.doi.org/10.1016/i.cie.2013.02.019

PONSIGNON, T.; LARS, M. Heuristic approaches for master planning in semiconductor manufacturing. Computers \& Operations Research, v. 39, p. 479-491, 2012. http://dx.doi.org/10.1016/j.cor.2011.06.009 
RIMAŠAUSKAS, M., BARGELIS, A. The development of the intelligent forecasting model for productivity index in manufacturing. Mechanika, v. 18 (3), p. 354-359, 2012.

http://dx.doi.org/10.5755/j01.mech.18.3.1879

SAITOU, K.; MALPATHAK, S.; QVAM, H. Robust design of flexible manufacturing systems using colored Petri net and genetic algorithm. Journal of Intelligent Manufacturing, v. 13 (5), p. 339-351, 2002. http://dx.doi.org/10.1023/A:1019920324813

SAMPAIO, R. F.; MANCINI, M. C. Estudos de Revisão Sistemática: Um guia para síntese criteriosa da evidência científica. Revista Brasileira de Fisioterapia, São Carlos, v. 11, n. 1, p. 83-89, 2007.

SAYGIN, C. Adaptive inventory management using RFID data. International Journal of Advanced Manufacturing Technology, v. 32 (9-10), p. 1045-1051, 2007. http://dx.doi.org/10.1007/s00170-006-0405-x

SILVA, T. F. G.; LEITE, M. S. A. A influência da gestão da capacidade na determinação do custo unitário de produção: um estudo de caso em uma empresa de embalagens plásticas flexíveis. Revista Produção Online, Florianópolis, SC, v.13, n. 3, p. 915-944, jul./set. 2013. http://dx.doi.org/10.14488/1676-1901.v13i3.1233

SOBREIRO, V. A.; ARAÚJO, P. H. S. L; NAGANO, M. S. Aplicação de sistemas dinâmicos na previsão de custos da produção. Rev. Eletrônica Produção \& Engenharia, v. 1, n. 1, p. 27-39, 2008.

STASIŠKIS, A.; ČIKOTIENĖ, D.; BARGELIS, A. Simulation of Products Classification System for Manufacturing Cost Forecasting. Mechanika, v. 17 (3), p. 300-305, 2011. http://dx.doi.org/10.5755/j01.mech.17.3.507

STOCKTON, D. J.; KHALIL, R. A.; MUKHONGO, L. M. Cost model development using virtual manufacturing and data mining: part II-comparison of data mining algorithms.

International Journal of Advanced Manufacturing Technology, v. 66, p. 1389-1396, 2013. http://dx.doi.org/10.1007/s00170-012-4416-5

TANG, C. S.; RAJARAM, K.; ALPTEKINOGLU, A.; OU, J. H. The benefits of advance booking discount programs: Model and analysis. Management Science, v. 50 (4), p. 465478, 2004. http://dx.doi.org/10.1287/mnsc.1030.0188

THOME, T.; FELIPE, L.; SUCLLA, N.; JOSE, A. Sales and operations planning: A research synthesis. International Journal of Production Economics, v. 138, p. 1-13, 2012. http://dx.doi.org/10.1016/i.ijpe.2011.11.027

THONEMANN, U. W. Improving supply-chain performance by sharing advance demand information. European Journal of Operational Research, v. 142 (1), p. 81-107, 2002. http://dx.doi.org/10.1016/S0377-2217(01)00281-8

TIACCI, L; SAETTA, S. Demand forecasting, lot sizing and scheduling on a rolling horizon basis. International Journal of Production Economics, v. 140, p. 803-814, 2012. http://dx.doi.org/10.1016/j.ijpe.2012.02.007

TIRKEL, I. Yield Learning Curve Models in Semiconductor Manufacturing. IEEE Transactions On Semiconductor Manufacturing, v. 26 (4), p. 564-571, 2013. http://dx.doi.org/10.1109/TSM.2013.2272017 
ÜLKÜ, S.; TOKTAY, L. B.; YÜCESAN, E. Risk ownership in contract manufacturing. Manufacturing and Service Operations Management, v. 9 (3), p. 225-241, 2007. http://dx.doi.org/10.1287/msom.1060.0141

VEIGA, C. P.; VEIGA, C. R. P.; VIEIRA, G. E.; TORTATO, U. Impacto financeiro dos erros na previsão empresarial: Um estudo comparativo entre Modelos Lineares e Redes Neurais. Revista Produção Online, Florianópolis, SC, v.12, n. 3, p. 629-656, jul./set. 2012. http://dx.doi.org/10.14488/1676-1901.v12i3.959

WANG, Q. Artificial neural networks as cost engineering methods in a collaborative manufacturing environment. International Journal of Production Economics, v. 109 (1-2), p. 53-64, 2007. http://dx.doi.org/10.1016/j.ijpe.2006.11.006

WU, S. D.; KEMPF, K. G.; ATAN, M. O.; AYTAC, B.; SHIRODKAR, S. A.; MISHRA, A. Improving New-Product Forecasting at Intel Corporation. Interfaces, v. 40 (5), p. 385-396, 2010. http://dx.doi.org/10.1287/inte.1100.0504

YIMER, A. D.; DEMIRLI, K. A genetic approach to two-phase optimization of dynamic supply chain scheduling. Computers \& Industrial Engineering, v. 58 (3), p. 411-422, 2010. http://dx.doi.org/10.1016/j.cie.2009.01.010

ZHANG, J.; SHOU, B.; CHEN, J. Postponed product differentiation with demand information update. International Journal of Production Economics, v. 141, p. 529-540, 2013. http://dx.doi.org/10.1016/.ijpe.2012.09.007

ZHANG, X.; PRAJAPATI, M.; PEDEN, E. A stochastic production planning model under uncertain seasonal demand and market growth. International Journal of Production Research, v. 49 (7), p. 1957-1975, 2011. http://dx.doi.org/10.1080/00207541003690074

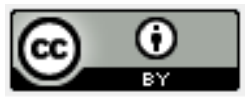

Artigo recebido em 18/10/2014 e aceito para publicação em 22/04/2015

DOI: $\underline{\text { http://dx.doi.org/ 10.14488/1676-1901.v15i2.1899 }}$ 